\title{
Pillule Dosage Form
}

National Cancer Institute

\section{Source}

National Cancer Institute. Pillule Dosage Form. NCI Thesaurus. Code C149758.

Solid preparation for homeopathic use, obtained from sucrose, lactose or other suitable excipients. Pillules may be prepared by impregnation of preformed pillules with a dilution or dilutions of homeopathic stocks or by progressive addition of these excipients and the addition of a dilution or dilutions of homeopathic stocks. Pillules are intended for oral or sublingual use. 